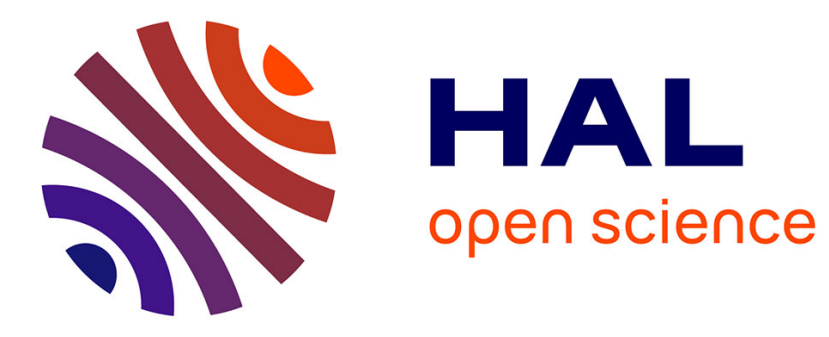

\title{
An Enhanced Sliding-Mode Control for a Pneumatic-Actuated Teleoperation System
}

Minh-Quyen Le, Minh Tu Pham, Mahdi Tavakoli, Richard Moreau

\section{To cite this version:}

Minh-Quyen Le, Minh Tu Pham, Mahdi Tavakoli, Richard Moreau. An Enhanced Sliding-Mode Control for a Pneumatic-Actuated Teleoperation System. 2011 IEEE IROS, Sep 2011, San Francisco, CA, United States. pp.659-664, 10.1109/IROS.2011.6048047 . hal-00619042

\section{HAL Id: hal-00619042 https://hal.science/hal-00619042}

Submitted on 27 Oct 2011

HAL is a multi-disciplinary open access archive for the deposit and dissemination of scientific research documents, whether they are published or not. The documents may come from teaching and research institutions in France or abroad, or from public or private research centers.
L'archive ouverte pluridisciplinaire HAL, est destinée au dépôt et à la diffusion de documents scientifiques de niveau recherche, publiés ou non, émanant des établissements d'enseignement et de recherche français ou étrangers, des laboratoires publics ou privés. 


\title{
An Enhanced Sliding-Mode Control for a Pneumatic-Actuated Teleoperation System
}

\author{
M.Q. Le, M.T. Pham, M. Tavakoli, R. Moreau
}

\begin{abstract}
This paper presents an enhanced sliding mode control for pneumatic master-slave teleoperation systems that are actuated by low-cost solenoid valves. A five-mode sliding control is incorporated into position-position, force-force, and force-position teleoperation architectures. While on/off valve pneumatic actuators have previously been modeled as having three discrete operating modes, an extension to five discrete control levels as proposed here helps to improve the actuator dynamic performance and reduce the switching activities of the valves. Stability and transparency analyses of the closed-loop teleoperation system are carried out. The proposed control design is experimentally tested on a singledegree-of-freedom pneumatic teleoperation system. Experimental results demonstrate high accuracies in terms of position and force tracking in the teleoperation system.
\end{abstract}

Index terms-Pneumatic actuator, on/off solenoid valve, sliding mode control, haptic teleoperation, stability.

\section{INTRODUCTION}

Teleoperation, which involves operating one or several robots remotely, has been an active research area over the past decade [1]. A teleoperation system typically involves a master robot handled by a human operator, and a slave robot operating at location far from the master robot.

The basic control requirements on a bilateral teleoperation system are related to its stability and transparency. Transparency corresponds to the quality of reproduction of the remote environment mechanical properties for the operator. Transparency in conjunction with stability can ensure successful completion of tasks in the teleoperation mode.

Traditionally, electrical direct-current (DC) motors are used as actuators in teleoperation systems as they are simple and capable of providing forces suitable for small haptic devices. In DC motor actuated haptic devices that require reflecting high output forces to the operator, the use of gears becomes necessary. This, however, will result in backlash, high inertia and discontinuity in output forces (cogging), which are undesirable as they distort the reflected forces. In addition, electrical motors are not compatible to applications that have extensive magnetic interference, (e.g., applications involving Magnetic Resonance Imaging; MRI) because they produce their own magnetic fields and contain ferromagnetic materials.

On the other hand, pneumatic actuators whose output force is a function of the compressed air pressure lead to

M.Q. Le, M.T. Pham, and R. Moreau are with the Laboratoire Ampère, UMR CNRS 5005, Université de Lyon, INSA-Lyon, F-69621 Villeurbanne Cedex, France (corresponding author's e-mail: minh-quyen.le@insalyon.fr). M. Tavakoli is with the Department of Electrical and Computer Engineering, University of Alberta, Edmonton, AB T6G2V4, Canada. higher force-to-weigh ratios than DC motors. Moreover, pneumatic actuators are inert to magnetic fields, which is crucial in applications such as robot-assisted surgery under MRI guidance [2]. Consequently, pneumatic actuators have found use in various applications of haptic teleoperation in recent years [3-5].

In this study, we investigate the development and control of a certain class of electro-pneumatic actuators in a teleoperation system. Generally, servo-valves, rather than solenoid (on/off) valves, are used to achieve high performances in pneumatic control. However, they are typically expensive due to the requirements of high-precision manufacturing. Therefore, in this paper, fast-switching on/off valves are used due to their advantages in terms of low cost and small size. One of the objectives of this paper is to show that good teleoperation transparency can be obtained with these inexpensive components as actuators of the teleoperation system.

The traditional control approach for pneumatic systems with solenoid valves involves using Pulse Width Modulation (PWM) [6], [7]. A main disadvantage of the PWM control is the chattering phenomenon caused by the high-frequency switching of the valves even in steady state [8].

To overcome the drawbacks of PWM-based control of solenoid valve actuated pneumatic devices, Nguyen et al. [9] proposed a nonlinear sliding mode controller, which controlled the actuator by modeling it as a discrete-input, three-mode system. In [10], we applied Nguyen's controller to the bilateral control problem of a pneumatic teleoperation system. In this paper, we will present an extension of Nguyen's controller and re-apply it to bilateral teleoperation control - this will result in superior performance compared to [9] and [10]. Specifically, we first extend the three-mode model used in [9] and [10] into a five-mode model. The two extra modes introduced in the new proposed scheme will offer more possibilities in terms of valve switching choices and will, therefore, improve the response of the system. Next, this new 5-mode sliding control law is used in a two-channel $(2 \mathrm{CH})$ bilateral teleoperation architecture with three different schemes: position-position, force-force, and force-position. These architectures are chosen due to their implementation simplicity and efficiency. Then, we provide analyses regarding the tracking performance, switching activity and closed-loop stability using a Lyapunov candidate function.

For simplicity, the master and the slave are identical and one-degree-of-freedom (DOF) pneumatic manipulators (with on/off actuation) in this study. It should be noted that this paper does not deal with compensating for time delays in a teleoperation system's communication channel. 
The paper is organized as follows. The modeling of the pneumatic manipulator composed of a cylinder and four solenoid valves is described in Section II. Section III presents a design of the 5-mode open-loop model of the master and slave manipulators. The sliding controller scheme for a closed-loop teleoperation system is then provided in Section IV. Section V shows experimental results that validate the proposed control laws. Finally, concluding remarks are given in Section VI.

\section{MODEL OF THE PNEUMATIC ACTUATOR}

As mentioned above, the master and the slave manipulators are identical, thus only one pneumatic robot is presented in this section. A schematic of the 1-DOF pneumatic actuation system is shown in Fig. 1.

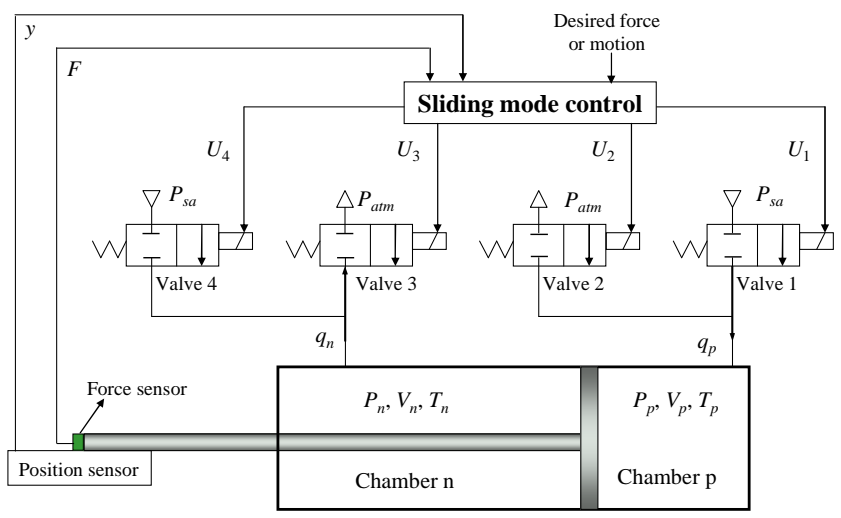

Fig. 1. Electro-pneumatic system with four valves

Assuming air is a perfect gas undergoing isothermal process, the behavior of the pressure inside each chamber of the cylinder can be expressed as

$$
\begin{aligned}
& \dot{P}_{p}=\frac{\gamma r T_{a}}{V_{p}(y)}\left(q_{p}\left(U_{1}, U_{2}, P_{p}\right)-\frac{P_{p}}{r T_{a}} S_{p} \dot{y}\right) \\
& \dot{P}_{n}=\frac{\gamma r T_{a}}{V_{n}(y)}\left(q_{n}\left(U_{3}, U_{4}, P_{n}\right)+\frac{P_{n}}{r T_{a}} S_{n} \dot{y}\right)
\end{aligned}
$$

where the subscripts $\mathrm{p}$ and $\mathrm{n}$ respectively denote chamber $\mathrm{p}$ and $\mathrm{n}, U_{1}, U_{2}, U_{3}$ and $U_{4}$ are the discrete control voltages ( 0 or 1 ) of valve 1 , valve 2 , valve 3 , and valve $4, y$ and $\dot{y}$ are the position $(\mathrm{m})$ and velocity $(\mathrm{m} / \mathrm{s})$ of the piston, $P_{p}$ and $P_{n}$ are the pressures $(\mathrm{Pa}), V_{p}$ and $V_{n}$ are the volumes of chambers $\left(\mathrm{m}^{3}\right), S_{p}$ and $S_{n}$ are the piston cylinder area $\left(\mathrm{m}^{2}\right), q_{p}$ and $q_{n}$ are the mass flow rates $(\mathrm{kg} / \mathrm{s}), T_{a}$ is the temperature of the supply air $(\mathrm{K}), r$ is the perfect gas constant $(\mathrm{J} /(\mathrm{kg} . \mathrm{K}))$ and $\gamma$ is the polytropic constant.

The mass flow rate characteristics of the on/off valves can be expressed as functions of the discrete control voltages and the pressures:

$$
\begin{aligned}
& q_{p}\left(U_{1}, U_{2}, P_{p}\right)=U_{1} q\left(P_{s a}, P_{p}\right)-U_{2} q\left(P_{p}, P_{a t m}\right) \\
& q_{n}\left(U_{3}, U_{4}, P_{n}\right)=U_{4} q\left(P_{s a}, P_{n}\right)-U_{3} q\left(P_{n}, P_{a t m}\right)
\end{aligned}
$$

where $P_{s a}$ and $P_{a t m}$ are the pressures of the supply air and atmosphere. The functions in (2) are given by a standard expression in which the mass flow rate of the valve is regulated by the air passage through an orifice:

$$
q\left(P_{u p}, P_{\text {down }}\right)=\left\{\begin{array}{l}
C_{v a l} P_{u p} \sqrt{T_{a t m} / T_{\text {app }}} \text { if } P_{\text {down }} / P_{u p} \leq 0.433 \text { (sonic) } \\
C_{v a l} P_{u p} \sqrt{T_{a t m} / T_{\text {tpp }}} \sqrt{1-\left(\frac{P_{d o w n} / P_{u p}-C_{r}}{1-C_{r}}\right)^{2}} \text { otherwise (subsonic) }
\end{array}\right.
$$

In the above, $C_{v a l}$ is the valve flow rate coefficient, $P_{u p}$ and $P_{\text {down }}$ are respectively the absolute upstream and downstream stagnation pressures of the valve, $T_{a t m}$ is the atmosphere temperature, and $T_{u p}$ is the upstream stagnation temperature.

Finally, the dynamics of the piston and the load are

$$
M \ddot{y}=S_{p} P_{p}-S_{n} P_{n}-b \dot{y}-F_{s t}+F_{\text {ext }}
$$

where $b$ is the viscous friction coefficient (N.s/m), $M$ is the moving load $(\mathrm{kg}), F_{s t}$ is the stiction force, and $F_{\text {ext }}$ is the external force $(\mathrm{N})$. For simplicity, the stiction force is assumed to be negligible.

\section{5-MODE OPEN-LOOP MODEL AND ENHANCED SLIDING CONTROL OF THE PNEUMATIC ACTUATOR}

\section{A. Controller mode selection}

In order to facilitate the control law design, a switching scheme for the four solenoid valves of Fig. 1 is defined so that each of the master and slave robots has the five modes of operation shown in Table I. Note that the three first modes are the three classical ones presented in [9], [10].

TABLE I: FIVE POSSIBLE CONTROL MODES

\begin{tabular}{cccccc}
\hline \hline & Mode 1 & Mode 2 & Mode 3 & Mode 4 & Mode 5 \\
\hline Ch. p & fills & exhausts & closed & fills & closed \\
\hline Ch. $\mathrm{n}$ & exhausts & fills & closed & closed & fills \\
\hline$u$ & 1 & -1 & 0 & 0.5 & -0.5 \\
\hline$U$ & {$\left[\begin{array}{llll}1 & 0 & 1 & 0\end{array}\right]$} & {$\left[\begin{array}{llll}0 & 1 & 0 & 1\end{array}\right]$} & {$\left[\begin{array}{llll}0 & 0 & 0 & 0\end{array}\right]$} & {$\left[\begin{array}{llll}1 & 0 & 0 & 0\end{array}\right]$} & {$\left[\begin{array}{llll}0 & 0 & 0 & 1\end{array}\right]$} \\
\hline \hline
\end{tabular}

In Table I, $U$ (the $4^{\text {th }}$ row) is the input voltage vector of the four valves, i.e., $U=\left[\begin{array}{llll}U_{1} & U_{2} & U_{3} & U_{4}\end{array}\right]$. The ' 0 ' state of each input voltage corresponds to a closed valve and the ' 1 ' state corresponds to an open valve. All the states where $U_{1}=U_{2}=$ 1 and $U_{3}=U_{4}=1$ are prohibited to avoid a bypass of the valves. Also, $u$ (the $3^{\text {rd }}$ row) is a newly introduced discrete control input that has five levels to match the five modes of operation. This new input can be chosen either as $u=$ $k \cdot \operatorname{sign}(s)$ or $u=-k \cdot \operatorname{sign}(s)$ where $k$ equals 1 or 0.5 , and $s$ is a sliding surface, which is a function of tracking error. Whether to choose the switching control $u$ as $k \cdot \operatorname{sign}(s)$ or as $k \cdot \operatorname{sign}(s)$ depends on the definition of $s$ and the open-loop system. As it will be shown later, this choice is crucial to ensuring the stability of the teleoperation system.

Note that mode 3 in Table I is used to "de-actuate" the piston when the tracking error is small enough. Mode 4 allows for moving the piston in the same direction (to the right in Fig. 1) as in mode 1 but with a slower dynamics (because in mode 4 the chamber $n$ is closed as opposed to exhausting as in mode 1; in both cases the chamber $\mathrm{p}$ will be filling). Mode 4 may be considered as an intermediate (or average) mode between modes 1 and 3, due to which the control vector $u$ is chosen as 0.5 (Table I). On the other hand, mode 5 whose control vector $u$ equals -0.5 is used to move the piston in the other direction and could be regarded as an 
intermediate level of actuation (and piston acceleration) between modes 2 and 3 .

\section{B. Open-loop models of master and slave}

Ignoring the stiction force in (4), the dynamics of the master and slave robots can be written as

$$
\begin{aligned}
& M \ddot{y}_{m}=S_{p} P_{p, m}-S_{n} P_{n, m}-b \dot{y}_{m}+f_{h} \\
& M \ddot{y}_{s}=S_{p} P_{p, s}-S_{n} P_{n, s}-b \dot{y}_{s}-f_{e}
\end{aligned}
$$

where $f_{h}$ and $f_{e}$ are the operator force exerted on the master and the environment force exerted on the slave, and $y_{m}$ and $y_{s}$ are the master and slave positions. Differentiating (5) and using (1)-(2), the dynamics of the master and slave manipulators are obtained after some manipulations as [9]:

$\dddot{y}_{m}=\left\{\begin{array}{l}\alpha_{m}+\beta_{m}^{+}+\dot{f}_{h} / M, u_{m}>0 \\ \alpha_{m}-\beta_{m}^{-}+\dot{f}_{h} / M, u_{m}<0 \\ \alpha_{m}+\dot{f}_{h} / M, u_{m}=0\end{array} \quad \dddot{y}_{s}=\left\{\begin{array}{l}\alpha_{s}+\beta_{s}^{+}-\dot{f}_{e} / M, u_{s}>0 \\ \alpha_{s}-\beta_{s}^{-}-\dot{f}_{e} / M, u_{s}<0 \\ \alpha_{s}-\dot{f}_{e} / M, u_{s}=0\end{array}\right.\right.$

where $u_{m}$ and $u_{s}$ denote the discrete control input as defined in Table I. In the above,

$$
\alpha_{i}=-\frac{b}{M} \ddot{y}_{i}-\frac{\gamma}{M}\left(\frac{S_{p}^{2} P_{p, i}}{V_{p}\left(y_{i}\right)}+\frac{S_{n}^{2} P_{n, i}}{V_{n}\left(y_{i}\right)}\right) \dot{y}_{i}
$$

When $u_{i}$ is positive, it might take two values, i.e., 1 or 0.5 , which corresponds to the modes 1 or 4 . Since $\beta_{i}^{+}$depends on the control choice of the valves, it can be written as

$$
\beta_{i}^{+}= \begin{cases}\gamma r T_{a}\left(\frac{S_{p}}{M} \frac{q\left(P_{s a}, P_{p, i}\right)}{V_{p}\left(y_{i}\right)}+\frac{S_{n}}{M} \frac{q\left(P_{n, i}, P_{a t m}\right)}{V_{n}\left(y_{i}\right)}\right) & , u_{i}=1 \\ \gamma r T_{a} \frac{S_{p}}{M} \frac{q\left(P_{s a}, P_{p, i}\right)}{V_{p}\left(y_{i}\right)} & , u_{i}=0.5\end{cases}
$$

Inversely, when $u_{i}$ is negative, it has two possible values, i.e., -1 or -0.5 , which relates to the control modes 2 or 5 . In this case, $\beta_{i}^{-}$is calculated as

$$
\beta_{i}^{-}= \begin{cases}\gamma r T_{a}\left(\frac{S_{p}}{M} \frac{q\left(P_{p, i}, P_{a t m}\right)}{V_{p}\left(y_{i}\right)}+\frac{S_{n}}{M} \frac{q\left(P_{s a}, P_{n, i}\right)}{V_{n}\left(y_{i}\right)}\right) & , u_{i}=-1 \\ \gamma r T_{a} \frac{S_{n}}{M} \frac{q\left(P_{s a}, P_{n, i}\right)}{V_{n}\left(y_{i}\right)} & , u_{i}=-0.5\end{cases}
$$

with $i=m$ or $s$ (for master or slave, respectively).

The operation of the 5-mode sliding controller is based on the following three principles:

1. In order to bring the system to the sliding surface $s=0$, which corresponds to perfect tracking performance, at steady state we define a neighbourhood of radius $\varepsilon<<1$ around zero. When $|s|$ is within the interval $[0, \varepsilon]$, mode 3 $(u=0)$ is used to conserve energy and somewhat reduce chattering.

2. To be able to switch between modes 1 and 4 or modes 2 and 5 , a threshold $\varepsilon_{1}$ is introduced where $\varepsilon_{1}>\varepsilon$. When $|\mathrm{s}|$ is within the interval $\left[\varepsilon, \varepsilon_{1}\right]$, either mode 4 or mode $5(u= \pm$ $0.5)$ is used to provide slower dynamics compared to modes 1 or $2(u= \pm 1)$, respectively. Still, the piston is actuated to move in the direction that minimizes $s$.

3. When $|\mathrm{s}|$ is within the interval $\left[\varepsilon_{1}, \infty\right]$, either mode 1 or mode $2(u= \pm 1)$ is used to provide fast dynamics, highly accelerating the piston in the direction that minimizes $s$.

In summary, we get our 5-mode sliding control law as

$$
u=\left\{\begin{array}{llr} 
\pm \operatorname{sign}(s) & \text { if } & |s| \geq \varepsilon_{1} \\
\pm 0.5 \operatorname{sign}(s) & \text { if } & \varepsilon<|s|<\varepsilon_{1} \\
0 & \text { if } & |s| \leq \mathcal{E}
\end{array}\right.
$$

\section{CLOSED-LOOP TELEOPERATION SYSTEM}

In this section, the five-mode sliding control is applied to a two-channel bilateral pneumatic teleoperation system with various different architectures (i.e., position-position, forceforce, and force-position). In the following, we provide analyses including tracking performance and closed-loop stability using a Lyapunov candidate function.

\section{A. Position error based (PEB) control}

A position-error-based, also called position-position, teleoperation system involves the simplest bilateral controller in which no force sensors are required. This architecture involves the transmission of two types of data between the master and the slave: position (or velocity) from the master to the slave and vice versa. The pneumatic-actuated PEB teleoperation system with our proposed sliding mode control is shown in Fig. 2.

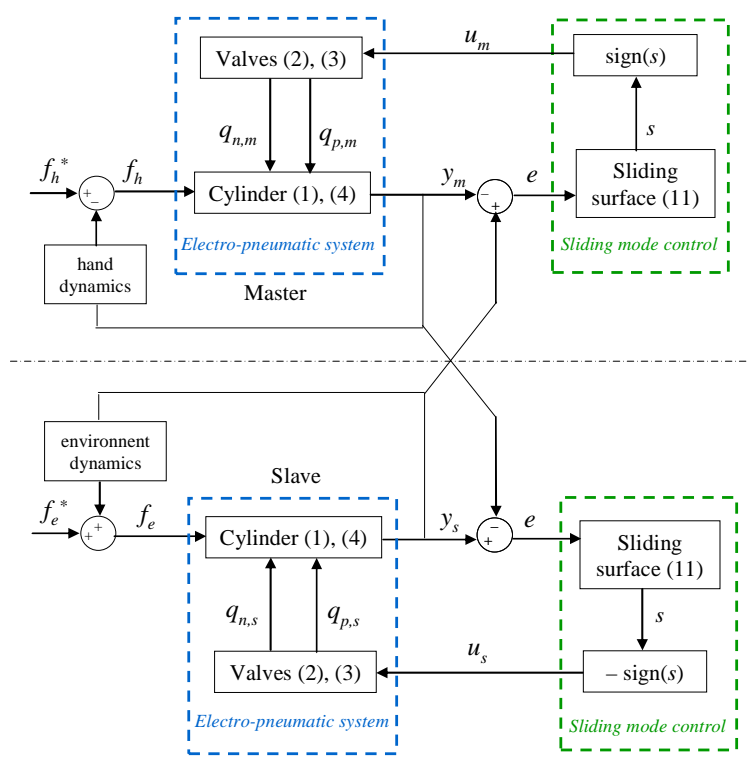

Fig. 2. Position-error-based approach with sliding mode control

In the PEB scheme, the sliding surface $s$ is defined as

$$
s=\ddot{e}+2 \xi \omega \dot{e}+\omega^{2} e
$$

where $e=y_{s}-y_{m}$ is the position error between the master and the slave and $\xi$ and $\omega$ are constant and positive parameters. The master and slave control laws $u_{m}$ and $u_{s}$ are defined as $u_{s}$ $=-u_{m}=-k \cdot \operatorname{sign}(s)$ where $k$ equals 1 or 0.5 .

Consider the following Lyapunov candidate function

$$
V=\frac{1}{2} s^{2}
$$

The sliding surface $s=0$ is reached within a finite time if the following condition is satisfied:

$$
\dot{V}=s \dot{s}<-\eta|s|
$$

for some constant $\eta>0$. Thus, from (11) and (13), we need

$$
s\left(\dddot{e}+2 \xi \omega \ddot{e}+\omega^{2} \dot{e}\right)<-\eta|s|
$$


Case 1: $s>0$. In this case, (14) becomes

$$
\left(\dddot{y}_{s}-\dddot{y}_{m}\right)+2 \xi \omega \ddot{e}+\omega^{2} \dot{e}<-\eta
$$

Since $s>0$, then $u_{s}<0$ and $u_{m}>0$. Therefore, the master and slave open-loop dynamics in (6) become

$$
\dddot{y}_{m}=\alpha_{m}+\beta_{m}^{+}+\dot{f}_{h} / M \quad, \quad \dddot{y}_{s}=\alpha_{s}-\beta_{s}^{-}-\dot{f}_{e} / M
$$

Substituting (16) in (15) results in the following condition

$$
\lambda-\left(\beta_{m}^{+}+\beta_{s}^{-}\right)<-\eta
$$

where

$$
\lambda=\alpha_{s}-\alpha_{m}-\left(\dot{f}_{h}+\dot{f}_{e}\right) / M+2 \xi \omega \ddot{e}+\omega^{2} \dot{e}
$$

Case $2: s<0$. Similar to case 1, we can easily deduce that

$$
\lambda+\left(\beta_{m}^{-}+\beta_{s}^{+}\right)>\eta
$$

where $\lambda$ is defined in (18).

Note that, from (8) and (9), $\beta_{i}^{+}$and $\beta_{i}^{-}$are positive, and can be made as large as desired by choosing a sufficiently large valve orifice $C_{v a l}$ in (3). Thus, to ensure that the conditions (17) and (19) are satisfied, we only need to show that $\lambda$ is bounded. For the demonstration of the boundedness of $\lambda$, the readers can refer to [10].

When $\lambda$ is bounded, the sliding condition in (13) is ensured, which implies that the position tracking error tends to zero (and that the overall system is stable).

Note that a drawback of the PEB method is that it does not guarantee a good transparency in term of force tracking. In order to improve the tracking performance, other schemes are proposed in the next subsections.

\section{B. Force error based (FEB) control}

A force-error-based, also called force-force, system is not commonly used in two-channel bilateral teleoperation since two force sensors are required and since position tracking is not good. However, compared to the PEB architecture, this architecture can improve the force tracking performance. The implementation of the proposed sliding mode control on the FEB system was schematically presented in [10].

Consider the control law $u_{s}=u_{m}=k \cdot \operatorname{sign}(s)$ where the sliding surface is defined as

$$
s=f_{h}-f_{e}
$$

Using the Lyapunov function (12), we need to show that the sliding condition (13) is satisfied. From the master and slave models (6), we can calculate $\dot{f}_{h}$ and $\dot{f}_{e}$ as

$$
\dot{f}_{h}=\left\{\begin{array}{l}
M\left(\dddot{y}_{m}-\alpha_{m}-\beta_{m}^{+}\right), u_{m}>0 \\
M\left(\dddot{y}_{m}-\alpha_{m}+\beta_{m}^{-}\right), u_{m}<0 \\
M\left(\dddot{y}_{m}-\alpha_{m}\right), u_{m}=0
\end{array}, \quad \dot{f}_{e}=\left\{\begin{array}{l}
M\left(-\dddot{y}_{s}+\alpha_{m}+\beta_{s}^{+}\right), u_{s}>0 \\
M\left(-\dddot{y}_{s}+\alpha_{m}-\beta_{s}^{-}\right), u_{s}<0 \\
M\left(-\dddot{y}_{s}+\alpha_{m}\right), u_{s}=0
\end{array}\right.\right.
$$

Case 1: $s>0$. In this case $u_{m}$ and $u_{s}$ are positive. From (13), we need

$$
\dot{s}=\dot{f}_{h}-\dot{f}_{e}=M\left(\dddot{y}_{m}-\alpha_{m}-\beta_{m}^{+}+\dddot{y}_{s}-\alpha_{s}-\beta_{s}^{+}\right)<-\eta
$$

It was shown in [10] that $\dddot{y}_{m}, \dddot{y}_{s}, \alpha_{m}$ and $\alpha_{s}$ are bounded. Thus, by choosing a valve with large orifice, $\beta_{m}^{+}$ and $\beta_{s}^{+}$can be made sufficiently large to satisfy (22). have

Case 2: $s<0$. In this case, $u_{m}$ and $u_{s}$ are negative. We

$$
\dot{s}=\dot{f}_{h}-\dot{f}_{e}=M\left(\dddot{y}_{m}-\alpha_{m}+\beta_{m}^{-}+\dddot{y}_{s}-\alpha_{s}+\beta_{s}^{-}\right)>\eta
$$

Similar to Case 1 , the stability of the system can be guaranteed by choosing a large enough value of $\beta_{m}^{-}$and $\beta_{s}^{-}$.

Finally, the force tracking error converges to zero and the overall system is stable. However, the FEB method does not guarantee a good position tracking performance. In order to overcome the PEB and FEB architecture drawbacks, we use the DFR scheme described in the following subsection.

\section{Direct force reflection (DFR) control}

A direct-force-reflection, also called force-position, system has advantages over the position-position and forceforce architectures. Compared to the PEB method, improvements in term of force tracking is achieved due to the measurement of the interaction force between the slave and the environment. Furthermore, its position tracking performance is better than the FEB case thanks to position information. For the implementation of the proposed sliding mode control on the DFR system, please refer to [10].

In this section, we use a Lyapunov function to prove the stability of the sliding-mode controlled DFR system. First, we will show the stability of the force-controlled master manipulator. Afterwards, we will show the stability of the position-controlled slave manipulator. However, the stability of the overall system is difficult to show due to the complexities introduced by using different sliding surfaces for the master and for the slave.

\section{1) Force convergence of the closed-loop master system}

The sliding surface $s_{m}$ and the Lyapunov function $V_{m}$ are defined as in (20) and (12), respectively. The controller $u_{m}$ is chosen to be similar to the FEB system in subsection IV.B.

Case 1: $s_{m}>0$. In this case, $u_{m}>0$. Using the expression of $\dot{f}_{h}$ in (21) and the definition of $s_{m}$ as in (20) we have

$$
\dot{s}_{m}=-\dot{f}_{e}+M \ddot{y}_{m}-M \alpha_{m}-M \beta_{m}^{+}
$$

To ensure the sliding condition (13), we need

$$
-\dot{f}_{e}+M \ddot{y}_{m}-M \alpha_{m}-M \beta_{m}^{+}<-\eta
$$

Similar to how it was demonstrated in [10], $\dot{f}_{e}, \dddot{y}_{m}$ and $\alpha_{m}$ can be shown to be bounded. Thus, the stability condition (25) is satisfied by choosing a large enough value for $\beta_{m}^{+}$.

Case 2: $s_{m}<0$. In this case, $u_{m}<0$. From (21) and (20) we need

$$
-\dot{f}_{e}+M \ddot{y}_{m}-M \alpha_{m}+M \beta_{m}^{-}>\eta
$$

Similar to Case 1 , it is possible to choose a large enough value of $\beta_{m}^{-}$to ensure the stability of the master robot.

Consequently, the sliding surface (the force tracking error) tends to zero, i.e., $f_{h}$ tends towards $f_{e}$.

\section{2) Position convergence of the closed-loop slave system}

The sliding surface $s_{s}$ and the Lyapunov function $V_{s}$ are defined as in (11) and (12) respectively. The controllers $u_{s}$ is chosen to be similar to the PEB system in subsection IV.A.

Case 1: $s_{s}>0$. In this case, $u_{s}<0$. The sliding condition (13) is equivalent to

$$
\left(\dddot{y}_{s}-\dddot{y}_{m}\right)+2 \xi \omega \ddot{e}_{s}+\omega^{2} \dot{e}_{s}<-\eta
$$

Using the expression of $\dddot{y}_{s}$ in (16) leads to 
where

$$
\varphi-\beta_{s}^{-}<-\eta
$$

$$
\varphi=\alpha_{s}-\dot{f}_{e} / M-\dddot{y}_{m}+2 \xi \omega \ddot{e}_{s}+\omega^{2} \dot{e}_{s}
$$

The straightforward reasoning described in [10] allows us to infer that $\varphi$ is bounded. Therefore, there exists a high value of $\beta_{s}^{-}$such as (28) is satisfied.

Case 2: $s_{s}<0$. In this case, $u_{s}>0$. Thus, we need

$$
\varphi+\beta_{s}^{+}>\eta
$$

where $\varphi$ is defined in (29)

This condition is achieved by choosing a large enough $\beta_{s}^{+}$.

Note that for both cases, the convergence of the sliding surface to zero is proved, so $x_{s}$ tends towards $x_{m}$.

\section{EXPERIMENTAL}

\section{A. Experimental setup}

In this section, experiments with a 1-DOF teleoperation system are reported. As illustrated in Fig. 3, the prototype consists of two identical master and the slave pneumatic manipulators. For more detail about the experimental description, please refer to [4]. The controller is implemented using a dSPACE board (DS1104), running at a sampling rate of $500 \mathrm{~Hz}$.

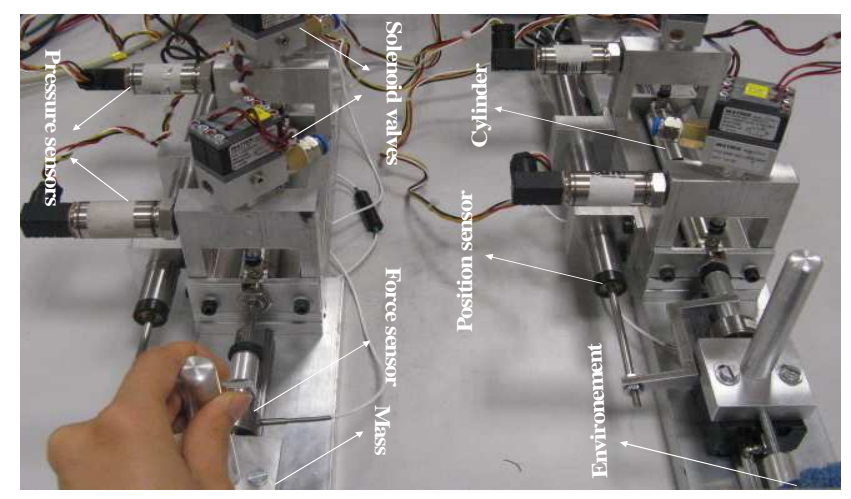

Fig. 3. Pneumatic master-slave teleoperation experimental setup

For the PEB and DFR systems, the sliding surface of the position-controlled slave is normalized as

$$
s=\frac{\ddot{e}}{\omega^{2}}+2 \frac{\xi}{\omega} \dot{e}+e
$$

This is because, in practice, it is easier if the sliding surface for position control is chosen to have the same dimension as positions as in (31) and not accelerations as in (11). The first derivative of the position error in (31) is computed through a backward difference method applied on the position signal followed by a second-order Butterworth filter with a cutoff frequency of $70 \mathrm{~Hz}$. The second derivative is computed in the same way from the filtered first-derivative signal.

For a good trade-off between the position tracking performance and the chattering problem, the parameters $\xi=$ 0.5 and $\omega=70 \mathrm{rad} / \mathrm{s}$ are chosen in practice.

In the experiments, the position and force thresholds $\varepsilon_{\mathrm{p}}$ and $\varepsilon_{\mathrm{f}}$ are respectively chosen equal to $0.5 \mathrm{~mm}$ and $0.1 \mathrm{~N}$ in order to achieve good tracking responses without causing too much switching of the valves.

\section{B. Experimental results}

Fig. 4 shows the master and the slave force and position tracking profiles in free space and in contact motion for the PEB teleoperation system. Fig. 5 and Fig. 6 illustrate the same profiles for the FEB and the DFR systems, respectively.

As illustrated in Fig. 4, the PEB system provides a good position tracking responses. However, the force response is not as good because no force sensor is used. On the other hand, the force tracking performance of the FEB system is much better, thanks to the knowledge of the force information. Nonetheless, the transparency of the position tracking deteriorates in FEB control - as it can be seen in Fig. 6, the slave's movement does not accurately track the master's movement.

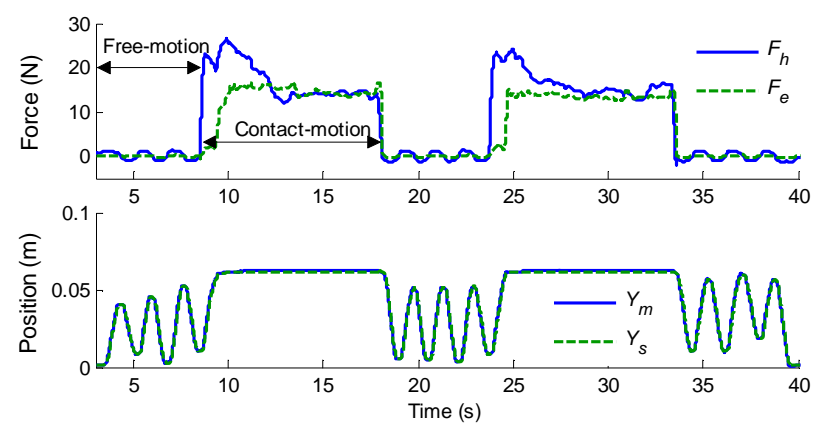

Fig. 4. Position and force profiles for the PEB teleoperation system
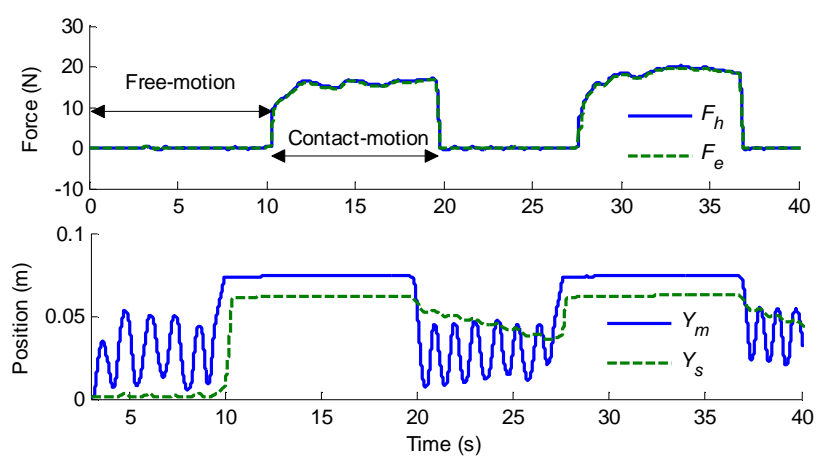

Fig. 5. Position and force profiles for the FEB teleoperation system

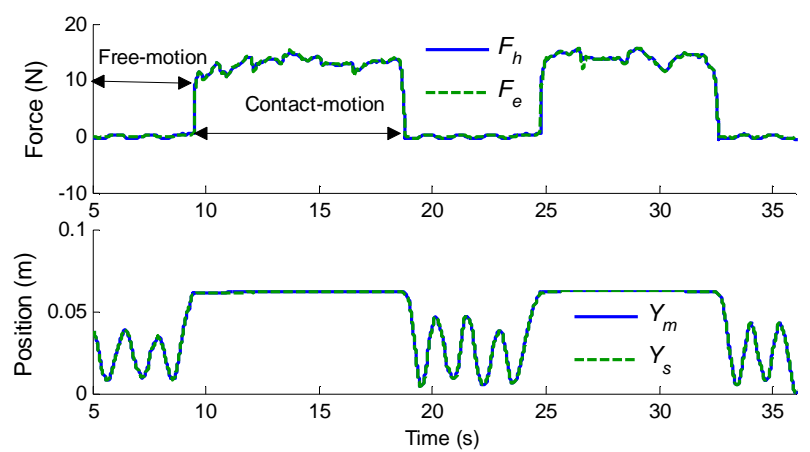

Fig. 6. Position and force profiles for the DFR teleoperation system

Interestingly, the DFR system in Fig. 6 provides an improvement in terms of position tracking response compared to the FEB system. It also displays a superior force tracking performance compared to the PEB, especially under contact motion where good quality force feedback is required. Thus, DFR control provides superior performance compared to both PEB and FEB control. Since the DFR 
system uses the measurement of slave/environment contact forces, the feeling of contact motion is highly realistic in our experiments. Therefore, the performance of the teleoperation system is improved significantly by feeding the operator with the slave/environment contact force. This result agrees with the previous theoretical work.

Among the three architectures, the DFR scheme seems to be a better choice to obtain a good transparency. Although the various teleoperation controllers have previously been compared from a performance perspective in the literature [11], [12], this is the first study to show that it is possible to achieve stability and satisfactory performance using manipulators actuated by low-cost switching on/off valves.

To show the benefits of the 5-mode control system teleoperation, we need to take the transparency analysis beyond only studying the force and position responses. Thus, a frequency analysis of the control signals (controller outputs) is carried out. In the following, due to the space restrictions, we only compare the spectra of the control signal $U_{1}$ (instead of $U_{1}, U_{2}, U_{3}$ and $U_{4}$ ) of the master side. Similar results can be observed for the other control signals. The frequency responses can be found by using the discrete fast Fourier transform fft command in Matlab. Fig. 7 shows the spectral analysis of the control signal $U_{1}$ obtained with the 5mode scheme and the 3-mode scheme of [10].
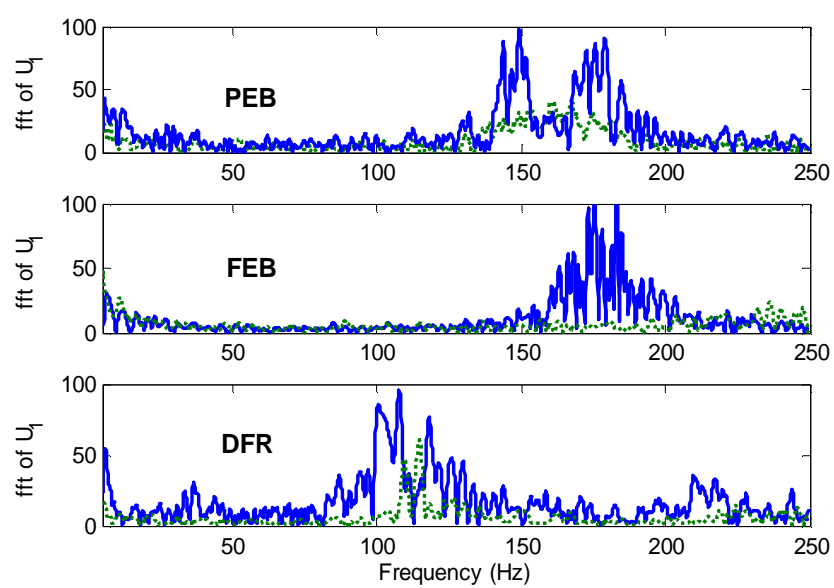

Fig. 7. Discrete Fourier transforms of the control signal $U_{1}$ for the master manipulator: 3-mode scheme (Solid), 5-mode scheme (Dotted)

As it can be observed, for all three architectures (PEB, FEB, and DFR), the magnitude $\left|U_{1}\right|$ of the spectra is lower in the 5-mode case than in the 3-mode case over almost all frequencies, and especially so at high frequencies. This shows that by using two additional modes, the on/off activity of the valves has been reduced, which allows to provide better tracking responses with smoother dynamics and less oscillations, particularly over high frequencies.

\section{CONCLUSION}

In this paper, pneumatic actuators with inexpensive solenoid valves are chosen for the development of a masterslave teleoperation system. To improve the dynamic performance and reduce the switching activities of the valves, a five-mode sliding control scheme has been used. Our study demonstrated that by increasing the number of the possible control actions for the valves, we can reduce the valves' switching activities, hence improving the valve's life times at no cost to teleoperation transparency. In order to evaluate the efficacy of the sliding mode approach, a comparison of the transparency and stability has been performed between three control architectures (PEB, FEB, and DFR) in a 2-channel bilateral teleoperation framework. The drawbacks of the PEB and FEB schemes in terms of less-than-ideal force or position tracking performance are analytically and experimentally demonstrated. Also, the DFR control scheme is shown to be highly transparent thanks to its used of force and position measurements.

The experiment results are encouraging for future work aimed at implementing the proposed sliding bilateral control on a direct-drive, multi-DOF, pneumatic-actuated teleoperation system. Another aspect of this work is to incorporate sliding mode control in more complex teleoperation architectures (e.g., three or four-channel methods) to improve the teleoperation transparency.

\section{REFERENCES}

[1] P. F. Hokayem and M. W. Spong, «Bilateral teleoperation: An historical survey », Automatica, vol. 42, n. 12, p. 2035-2057, 2006.

[2] Ningbo Yu, C. Hollnagel, A. Blickenstorfer, S. S. Kollias, and R. Riener, «Comparison of MRI-Compatible Mechatronic Systems with Hydrodynamic and Pneumatic Actuation», IEEE/ASME Transactions on Mechatronics, vol. 13, $\mathrm{n}^{\mathrm{o}}$. 3, p. 268-277, 2008.

[3] V. Durbha and P. Y. Li, «Passive bilateral tele-operation and human power amplification with pneumatic actuators », Proceedings of ASME Dynamic Systems and Control Conference, p. 1-8, 2009.

[4] M. Q. Le, M. T. Pham, M. Tavakoli, and R. Moreau, «Development of a hybrid control for a pneumatic teleoperation system using on/off solenoid valves», IEEE International Conference on Intelligent Robots and Systems, p. 5818-5823, 2010.

[5] K. Tadano and K. Kawashima, «Development of a master slave system with force sensing using pneumatic servo system for laparoscopic surgery », IEEE International Conference on Robotics and Automation, vol. 18, p. 947-952, 2007.

[6] M. C. Shih and M. Ma, «Position control of a pneumatic rodless cylinder using fuzzy PWM control method », Mechatronics, vol. 41, $\mathrm{n}^{\circ}$. 2, p. 241-253, 1998.

[7] M. Taghizadeh, A. Ghaffari, and F. Najafi, «Improving dynamic performances of PWM-driven servo-pneumatic systems via a novel pneumatic circuit », ISA Transactions, vol. 48, nº. 4, p. 512-518, 2009.

[8] M. Q. Le, M. T. Pham, R. Moreau, J. P. Simon, and T. Redarce, «Force Tracking of Pneumatic Servo Systems using on/off Solenoid Valves based on a Greedy Control Scheme », ASME Journal of Dynamic Systems, Measurement, and Control, vol. 133, n ${ }^{\circ} .5,2011$, In press.

[9] T. Nguyen, J. Leavitt, F. Jabbari, and J. E. Bobrow, « Accurate sliding-mode control of pneumatic systems using low-cost solenoid valves », IEEE/ASME Transactions on mechatronics, vol. 12, $\mathrm{n}^{\circ} .2$, p. 216-219, 2007.

[10] M. Q. Le, M. T. Pham, M. Tavakoli, and R. Moreau, «Sliding Mode Control of a Pneumatic Haptic Teleoperation System with on/off Solenoid Valves», IEEE International Conference on Robotics and Automation (ICRA), p. 874-879, 2011.

[11] M. Tavakoli, A. Aziminejad, R. V. Patel, and M. Moallem, «Enhanced Transparency in Haptics-Based Master-Slave Systems », American Control Conference, p. 1455-1460, 2007.

[12] H. Y. K. Lau and L. C. C. Wai, « Implementation of position-force and position-position teleoperator controllers with cable-driven mechanisms ", Robotics and Computer-Integrated Manufacturing, vol. 21, nº. 2, p. 145-152, 2005. 